\title{
Laser-Induced Forward Transfer of Pre-Machined Donor Films
}

\author{
K.S.Kaur ${ }^{1}$, M.Feinaeugle ${ }^{1}$, D.P.Banks ${ }^{1}$, J.Y. Ou ${ }^{1}$, F.Di Pietrantonio ${ }^{2}$, E. Verona ${ }^{2}$, C.L.Sones ${ }^{1}$, R.W.Eason ${ }^{1}$ \\ ${ }^{1}$ Optoelectronics Research Centre, University of Southampton, Southampton SO17 IBJ, UK. \\ ${ }^{2}$ Institute of Acoustics and Sensors- CNR 10000133 Rome - Italy \\ kak@orc.soton.ac.uk
}

\begin{abstract}
Micro-pellets of $\mathrm{ZnO}$ with a diameter of 5-10 $\mu \mathrm{m}$ and extremely smooth edges have been printed using laser-induced forward transfer from $1 \mu \mathrm{m}$ thick films, that had been prepatterned using focussed ion beam (FIB) machining.

OCIS codes: (140.3390) Laser materials processing; (140.7090) Ultrafast lasers; (220.4000) Microstructure fabrication; Laser-Induced Forward Transfer.
\end{abstract}

\section{Introduction}

Laser-induced forward transfer (LIFT) is a versatile direct write technique for spatially selective printing of a wide range of materials [1]. However the major drawback of the conventional LIFT method is that the material to be printed (the donor) is directly exposed to the incoming laser pulse and this can lead to surface damage, complete melting or disintegration during the transfer process. To avoid this problem a sacrificial layer (metallic or polymer) can be introduced between the donor and the carrier substrate in a complementary LIFT technique called the dynamic release layer (DRL)-LIFT technique [2]. However there are issues of post transfer contamination with the residual DRL [3], or chemical and thermal sensitivity of the DRL material [4]. Most importantly the basic problem of the ripping and shearing of the donor film at the boundary of the illuminated zone still persists in DRL-LIFT that result in deposits with rough edges, as well as unwanted debris present on the receiver. This shearing around the edges also limits the maximum thickness of the donor material that can be printed using LIFT.

In this paper we present a new technique that not only prints solid donors in an intact form but should also extend the thickness limitation to beyond the current $\sim \mu \mathrm{m}$ level [5], without using any DRL. The idea is to premachine the donor material prior to LIFTing to encourage transfer of predetermined shapes in intact and solid form. In our case a focussed ion beam (FIB) was used to pre-machine the donor films. Zinc oxide (ZnO), an environmental-friendly lead free piezoelectric was chosen as the trial donor material for these FIB assisted LIFT experiments.

\section{Experiments and results}

Donor samples were prepared by sputtering $1 \mu \mathrm{m}$ thick films of $\mathrm{ZnO}$ on top of quartz substrates at a pressure of 3 mTorr and a temperature of $200^{\circ} \mathrm{C}$. FIB machining was used to etch circular features into these $1 \mu \mathrm{m}$ thick sputtered $\mathrm{ZnO}$ films up to a depth of $0.8 \mu \mathrm{m}$ with $5 \mu \mathrm{m}$ and $10 \mu \mathrm{m}$ diameters and centre-to-centre separation of $50 \mu \mathrm{m}$. For comparison $1 \mu \mathrm{m}$ thick $\mathrm{ZnO}$ films on top of triazene polymer (TP) [4], as a DRL and $1 \mu \mathrm{m}$ thick ZnO samples without any prior machining were also prepared and LIFTed. Single pulses (800 nm, $150 \mathrm{fs})$ from a commercial Ti:sapphire femtosecond laser were used to print micro-pellets of $\mathrm{ZnO}$ onto silicon and plastic substrates (the receiver) at a donor-receiver separation of $1 \mu \mathrm{m}$, using Mylar spacers. All the experiments were performed under a background pressure of $10^{-1} \mathrm{mbar}$.

Figure 1(a) shows SEM micrographs of ZnO deposits printed from a $1 \mu \mathrm{m}$ thick $\mathrm{ZnO}$ film using $360 \mathrm{~nm}$ thick TP as the DRL. The irregular shapes of the deposits were due to the decomposition of the TP layer while depositing the upper $\mathrm{ZnO}$ layer. Cracks were clearly visible in the LIFTed deposits, and as the film was weaker around the cracks LIFTing preferentially occurred by rupture along the cracks leading to non-circular deposits. The deposits printed from a $1 \mu \mathrm{m}$ thick $\mathrm{ZnO}$ donor without any pre-machining were in general splashy, with considerable amounts of debris and with very rough and ill-defined edges and irregular shapes. The poor edge quality of a typical deposit is shown in fig. 1 (b).

However, the deposits from a pre-machined donor exhibited extremely good quality both in terms of surface uniformity and edge smoothness. Under the optimum conditions for LIFTing, there was no trace of any residual debris surrounding the transferred pellets. Figure 2 (a) and 2 (b) show the SEM images of $10 \mu \mathrm{m}$ and $5 \mu \mathrm{m} \mathrm{ZnO}$ pellets respectively printed from a pre-machined donor. Figure 2(c) shows an SEM image of the edge quality of a printed $10 \mu \mathrm{m} \mathrm{ZnO}$ pellet, and this degree of smoothness was routinely seen in all SEM pictures taken. The striking difference in the quality of the deposits obtained clearly shows the great potential of this technique for printing thick and fragile donors in solid and intact format. 

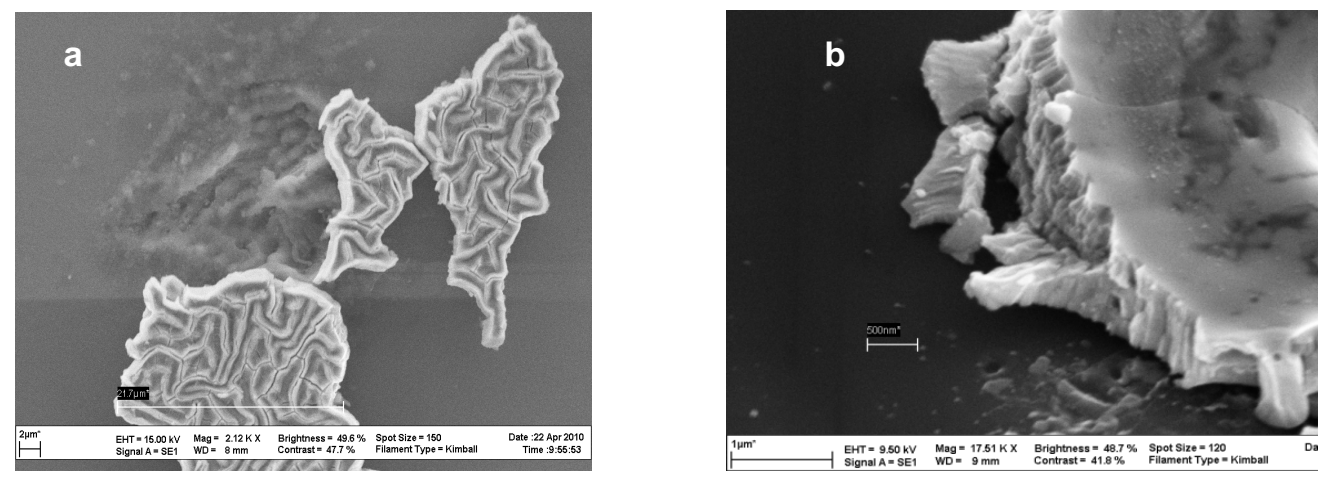

Fig. 1. (a) Shows the SEM image of $\mathrm{ZnO}$ deposits printed using TP as DRL; (b) shows rough edges of a typical $\mathrm{ZnO}$ deposit printed from a non-machined donor film.
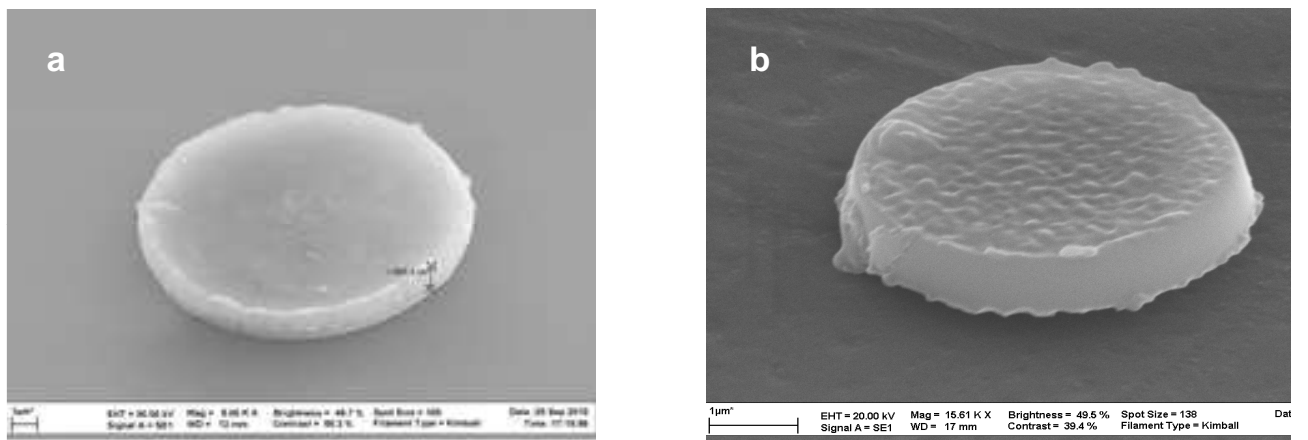

Fig. 2 (a) and (b). SEM micrographs of $10 \mu \mathrm{m}$ and $5 \mu \mathrm{m} \mathrm{ZnO}$ pellets printed onto Si and plastic receiver respectively from a pre-machined 1 $\mu \mathrm{m}$ thick donor film.

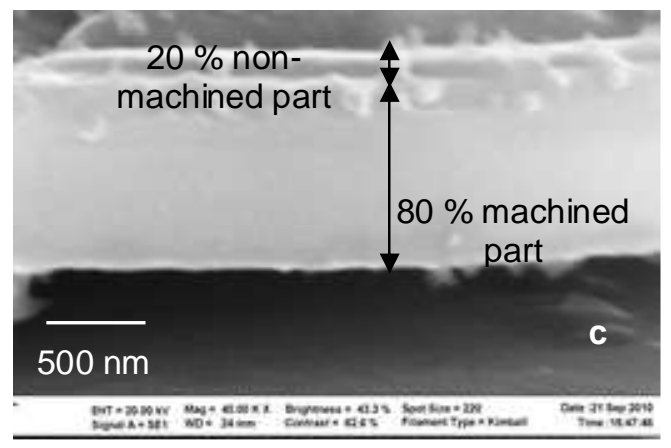

Fig. 2(c). Magnified SEM image of the extraordinarily smooth edge of one of the deposits printed from the pre-machined $\mathrm{ZnO}$ donor.

\section{Conclusions}

Debris free micro-pellets of $\mathrm{ZnO}$ of excellent quality with extremely smooth and uniform edges have been printed from pre-machined donor films using an FIB-assisted LIFT technique.

\section{References}

[1] J. Bohandy, B.F. Kim, F.J. Adrian, "Metal deposition from a supported metal film using an excimer laser,”J. Appl. Phys. 60, 1538 (1986).

[2] W.A. Tolbert, I.-Y.S. Lee, M.M. Doxtader, E.W. Ellis, D.D. Dlott, "High-speed color imaging by laser ablation transfer with a dynamic release layer: fundamental mechanisms," J. Imaging Sci. Technol. 37, 411 - 421 (1993).

[3] T.Smausz, B. Hopp, G. Kecskemeti, Z. Bor, "Study on metal microparticle content of the material transferred with absorbing film assisted laser induced forward transfer when using silver absorbing layer," Appl. Surf. Sci. 252, 4738-4742 (2006).

[4] R Fardel, P. Feurer, T. Lippert, M. Nagel, F. Nuesch, A. Wokaun, "Laser ablation of aryltriazene photopolymer films: Effects of polymer structure on ablation properties," Appl. Surf. Sci., 254, 13321337 (2007).

[5] K.S.Kaur, R.Fardel, T.C.May-Smith, M.Nagel, D.P.Banks, C.Grivas, T.Lippert, R.W.Eason, "Shadowgraphic studies of triazene assisted laser-induced forward transfer of ceramic thin films,"J. Appl. Phys. 105113119 (2009). 